Meta

Journal des traducteurs

Translators' Journal

\title{
Formé sur le tas
}

\section{Shlomo Gitai}

Volume 38, numéro 4, décembre 1993

Le Je du traducteur

The $I$ of the Translator

URI : https://id.erudit.org/iderudit/002923ar

DOI : https://doi.org/10.7202/002923ar

Aller au sommaire du numéro

\section{Éditeur(s)}

Les Presses de l'Université de Montréal

\section{ISSN}

0026-0452 (imprimé)

Découvrir la revue

\section{Citer cet article}

Gitai, S. (1993). Formé sur le tas. Meta, 38(4), 674-676.

https://doi.org/10.7202/002923ar

Ce document est protégé par la loi sur le droit d'auteur. L'utilisation des services d'Érudit (y compris la reproduction) est assujettie à sa politique d'utilisation que vous pouvez consulter en ligne.

https://apropos.erudit.org/fr/usagers/politique-dutilisation/
Cet article est diffusé et préservé par Érudit.

Érudit est un consortium interuniversitaire sans but lucratif composé de l’Université de Montréal, l'Université Laval et l'Université du Québec à Montréal. Il a pour mission la promotion et la valorisation de la recherche. https://www.erudit.org/fr/ 


\section{FORMÉ SUR LE TAS}

SHLOMO GiTAI

Université Bar-Ilan, Ramat-Gan, Israël

Imaginez un petit café au centre de Jérusalem, en 1959. Pas grand-chose, à l'époque, mais tout de même plein de monde dans la soirée. Deux hommes s'installent à un guéridon au centre de la salle. Et puis, subitement, ils commencent à parler, les deux à la fois, à haute voix, sans arrêt, avec volubilité. Un couple à la table voisine découvre, avec surprise, que les deux orateurs ne parlent pas la même langue. Il se lève pour regarder. Bientôt un autre couple se joint à eux. Cinq minutes plus tard, tout le café est là, à admirer le spectacle. Et les deux fous, de continuer leur palabre, toujours plus fort, toujours plus vite, toujours dans deux langues différentes.

Brusquement le silence s'est fait, Adam Richter s'est tu, je me tais aussi. Passant du français à l'hébreu, il me dit: «Ça va, je vous engage» et moi, passant de l'espagnol à l'hébreu, je l'en remercie.

C'est ainsi que j'ai commencé ma carrière d'interprète. Après cette épreuve, publique si j'ose dire, Richter, père de l'interprétation simultanée en Israël, décédé depuis lors, me soumit à une période d'entraînement d'une semaine environ, puis m'envoya faire mes débuts au Congrès sioniste. Il devait se dire que les débats, plutôt assoupissants, ne méritaient pas mieux. En tout cas, il est certain que j'ai été formé sur le tas. Richter m'avait recruté au service des informations de la radio israélienne, où je travaillais depuis un certain temps.

Après ces aveux, serai-je radié de l'AIIC, honni par mes camarades de travail et renvoyé de mon poste d'enseignant à l'École d'interprètes de l'Université Bar-Ilan? J'espère bien que non. Avec plus de trente années de métier, même parti de zéro, j'aurais fini par apprendre quelque chose. Cependant, la question se pose quant à la validité de la formation sur le tas d'un interprète. Je ne pense pas pouvoir donner de réponse universelle, je peux toutefois présenter mon propre cas, dont chacun tirera les conclusions qu'il voudra.

Ce qui m'a permis de me lancer ainsi dans l'interprétation pratiquement sans préparation préalable, c'est tout d'abord, à mon avis, le fait d'avoir appris plusieurs langues à la fois dès ma plus tendre enfance. Mon cas, que je résumerai brièvement, est typique de bien des familles juives.

Mes ancêtres, chassés d'Espagne par les Rois catholiques, s'installèrent en Turquie au $\mathrm{XV}^{\mathrm{e}}$ siècle, où ils continuèrent à parler un castillan médiéval — plus ou moins déformé au cours des siècles - que les spécialistes appellent «judéo-espagnol». C'était la seule langue que ma grand-mère connaissait et les histoires qu'elle me racontait dans cette langue ont meublé les rêves de mon enfance. Mes parents, nés tout les deux à Istanbul, reçurent leur éducation dans les écoles de l'Alliance israélite universelle; aussi s'exprimaient-ils surtout en français, et c'était en fait la langue que nous parlions tous à la maison. Mon père, établi à Barcelone, séjournait souvent à Paris, avec sa famille, et c'est à cheval entre ces deux villes que s'est passée mon enfance. À Barcelone, j'appris l'espagnol à l'école et le catalan dans la rue. Aujourd'hui, établi en Israël depuis près de quarante ans, je parle l'hébreu avec ma femme et mes enfants et, entre-temps, j'ai aussi appris l'anglais. Remarquez, en passant, que chaque génération parle une langue différente.

La connaissance de plusieurs langues est très courante en Israël et nombreux sont ici ceux qui, outre l'hébreu, parlent le yiddish, le polonais et le russe, ou bien l'arabe, 
l'iranien et le turc ou, comme l'une de mes amies, le suédois, le finlandais, le français, l'italien, et j'en passe. Sommes-nous tous, dans ce pays, des interprètes en puissance?

«Pensez-vous, me répondra-t-on, votre catalan, appris dans la rue, ne vaut rien, votre français est médiocre, votre anglais est minable, le judéo-espagnol n'est pas une langue, à peine un jargon, et votre espagnol, qu'en reste-t-il, après quarante années de séjour hors d'Espagne? Tous ces Israéliens qui, comme vous, parlent ou prétendent parler cinq ou six. langues, n'en connaissent en fait aucune et feraient mieux de laisser la traduction et l'interprétation à des professionnels, dûment formés dans les écoles spécialisées.»

C'est probablement vrai, c'est pourquoi il faut de plus, à mon avis, être doué pour l'interprétation, comme on est doué pour la peinture, la musique ou les mathématiques. On dira qu'à la rigueur cette règle s'applique à n'importe quelle profession. Il est vrai que, sans talent, un interprète, même formé dans les meilleures écoles, n'ira pas loin; formé uniquement sur le tas, il n'ira nulle part. J'estime, d'ailleurs, qu'il n'y a aucun rapport entre la connaissance des langues et la faculté d'interpréter, ce qui fait qu'à la limite on pourrait trouver, j'imagine, des interprètes-nés, ayant un talent extraordinaire pour l'interprétation, mais qui, ne connaissant qu'une seule langue, ne pourraient le manifester. C'est là un sujet de recherche que j'offre aux spécialistes.

Être doué, connaitre des langues, est-ce suffisant pour faire un bon interprète formé sur le tas? J'estime que non. Il faut en outre avoir ce que, faute d'une expression plus scientifique, $\mathrm{j}$ 'appellerai des antennes de traducteur, des antennes toujours en quête de nouveaux termes, que la mémoire (ou le carnet) enregistreront pour en chercher aussitôt que possible l'équivalent dans les autres langues de l'interprète. Ce souci constant de repérer et d'enregistrer des mots nouveaux, des expressions peu courantes, des équivalences de termes, est de la plus grande importance pour tout interprète, mais, pour ceux qui comme moi n'ont pas été formés dans les écoles, il est vital, et remplace une partie de ce qu'un bon programme d'études aurait dû fournir.

Il y a aussi une condition importante à remplir pour qu'un enfant, élevé comme moi dans un milieu multilingue, puisse devenir un interprète digne de ce nom: il faut à tout prix, constamment, obstinément, se refuser à tout mélange de langues. Habitué, par exemple, à utiliser le mot français fougère, imaginez que dans une phrase en espagnol le terme équivalent (helecho) vous fasse défaut; quoi de plus simple, dans ces conditions, que d'utiliser le terme français, si tout le monde autour de vous le comprend. C'est une tendance très naturelle, mais aussi très pernicieuse pour l'interprète en herbe. Malheureusement, elle se retrouve souvent chez les groupes d'immigrants, qui ont vite fait de se créer ainsi des sabirs, incompréhensibles au commun des mortels. En Israël, on entend souvent parler des langues étrangères - anglais, français, espagnol ou autres - copieusement truffées de mots hébraïques de la vie quotidienne. Résister à cette tendance demande parfois un effort très sérieux. J'en sais quelque chose, il y a une quarantaine d'années que je le fais.

Ce qui m'a aussi beaucoup aidé lors de mes débuts dans l'interprétation, c'est une expérience non négligeable de la traduction écrite, ce qui est assez normal. Mais il y a lieu de se demander comment je suis devenu traducteur, de nouveau sans formation préalable dans ce domaine. En fait, $\mathrm{j}$ 'avais une formation assez valable : $\mathrm{j}$ 'avais surtout fait de la traduction technique, fort des trois années d'études en sciences que j'avais faites avant de venir $m$ 'installer en Israël. Dans la traduction technique, on pardonne facilement le manque de savoir-faire du traducteur, ou un style inélégant, si, en revanche, il est capable de comprendre ce qu'il traduit, sans commettre de bévues épouvantables. L'idéal serait, à mon avis, d'arriver à combiner les études dans les deux domaines, sciences et traduction, en vue de former des traducteurs techniques qualifiés, mais, pour autant que je sache, aucune école de traducteurs n'a encore introduit de programme de cette nature. C'est un effort qui mériterait d'être fait. 
J'ai donc fait pendant longtemps - plus de vingt-cinq années - de la traduction technique à côté de l'interprétation, ce qui m'a permis de vivre tant bien que mal. En Israël, aucune de ces deux professions ne permet de faire fortune. J'ai dit, déjà, que la majorité des Israéliens parlent couramment deux ou trois langues. Par conséquent, tout bon Israélien estime que la traduction, loin d'être un art ou une discipline compliquée et difficile à maitriser, est un jeu d'enfants. «Interprète, traducteur? - vous dira-t-on avec une pointe d'ironie c'est quoi, ça? Tous mes amis parlent trois langues, ma grand-mère en parle cinq, ils ne se font pas payer pour ça. Je vous payerai comme nos dactylos, qui peuvent taper une lettre à la machine en trois langues.» II n'est pas rare de voir dans ce pays des offres d'emplois de «dactylo-traductrice». Autrement dit, le métier de traducteur ou d'interprète est partout ingrat, en Israël il est, de plus, sous-estimé, pour ne pas dire méprisé.

Cependant, à côté des émigrants et fils ou petits-fils d'émigrants, qui parlent encore les langues de l'ancien pays, on trouve un nombre croissant d'Israéliens nés dans le pays, qui ne connaissent que l'hébreu. Leur problème est au fond le même que celui des Français, des Australiens, des Mexicains, etc., qui, normalement, ne parlent eux aussi qu'une seule langue. Le problème est moins grave lorsqu'on pense à une langue à grande diffusion, mais que font ceux qui ne parlent que le géorgien, le tamoul ou le papiamento? Ils apprennent des langues étrangères depuis leur plus jeune âge, c'est évident. Ce devrait être aussi le cas des jeunes Israéliens dont les parents ou les grands-parents sont nés eux aussi en Israël et ne parlent pas d'autres langues à la maison. Cependant, dans ce pays ou tout le monde affirme connaître quatre langues, les jeunes autochtones ont un mal fou à en apprendre une deuxième. Est-ce dû au fait que l'hébreu s'écrit en des caractères différents de ceux des langues européennes, ou bien qu'on l'écrit sans voyelles, ou qu'on le lit à rebours? Voilà encore un sujet qui mériterait une recherche sérieuse, et que j'offre aux spécialistes.

En tout cas, l'immigration est une mauvaise école de langues, on n'y apprend en général que le jargon de la rue, souvent agrémenté d'un accent étranger. Israël, pays d'immigrants par excellence, ne fait pas exception à la règle. Heureusement pour nous, l'hébreu moderne (l'israélien, dirais-je, comme on dit l'américain) est une langue en pleine évolution, dont les règles sont méconnues ou volontairement ignorées de la plupart d'entre nous, et dont l'accent n'est pas encore fixé. Ceci permet à un immigrant comme moi, qui dans un autre pays serait récusé à cause de son accent étranger ou de ses tournures de phrase bizarres, d'être accepté comme interprète vers la langue du pays.

J'ai raconté plus haut comment j'ai commencé ma carrière d'interprète. En fait ce n'est pas tout à fait vrai. En 1939, peu après la déclaration de guerre, tous les adultes de Paris avaient reçu des masques à gaz, mais comme il n'existait pas pour les enfants de modèle assurant une protection complète, tous les enfants de la ville avaient été évacués à la campagne. C'est ainsi que j'ai débarqué, par une nuit noire comme l'encre, dans un village minuscule, où d'ailleurs, cinq ans plus tard, les Américains devaient débarquer eux aussi, pour libérer la France (encore aujourd'hui, l'endroit s'appelle Omaha Beach). Or, en 1939, il y avait en France peu de main-d'œuvre disponible, et beaucoup de réfugiés de la guerre d'Espagne. Le maire du petit patelin avait donc engagé quelques braves femmes espagnoles, d'un camp de réfugiés voisin, pour les travaux de ménage dans le camp des enfants. Incapable de se faire comprendre de son personnel, il a cherché - et trouvé - un interprète. J'ai même été payé: un sachet de bonbons. 